\title{
A Coupled Multigrid Method for Nonconforming Finite Element Discretizations of the 2D-Stokes Equation
}

\author{
V. John and L. Tobiska, Magdeburg \\ Received January 19, 1999; revised September 13, 1999
}

\begin{abstract}
This paper investigates a multigrid method for the solution of the saddle point formulation of the discrete Stokes equation obtained with inf-sup stable nonconforming finite elements of lowest order. A smoother proposed by Braess and Sarazin (1997) is used and $L^{2}$-projection as well as simple averaging are considered as prolongation. The $\mathrm{W}$-cycle convergence in the $L^{2}$-norm of the velocity with a rate independently of the level and linearly decreasing with increasing number of smoothing steps is proven. Numerical tests confirm the theoretically predicted results.
\end{abstract}

AMS Subject Classifications: 65N12, 65N22, 65N55.

Key Words: Nonconforming finite element discretizations, coupled multigrid methods, Braess-Sarazin smoother, Stokes equation.

\section{Introduction}

In a comparative study of solvers for the incompressible Navier-Stokes equation by means of the nonconforming $P_{1} / P_{0}$-finite element discretization, [15], coupled multigrid methods have been proven to be superior to pressure Schur complement type schemes, e.g. SIMPLE. In [16], the performance of different smoothers within coupled multigrid methods have been tested and compared for the solution of the Navier-Stokes equation. Braess-Sarazin-type smoothers exhibit good smoothing properties. Although one smoothing step is computationally expensive, coupled multigrid methods using Braess-Sarazin-type smoothers have been proven as useful solvers for incompressible flow problems. However, a theoretical support of these methods in the full nonlinear case is still missing. Therefore, in this paper, we study the Stokes equations as a simple model of Computational Fluid Dynamics.

Nonconforming finite element approximations for incompressible flows are attractive since they easily fulfil the discrete version of the Babuška-Brezzi condition. Another advantage of using nonconforming finite elements is that the unknowns are associated with the element faces such that each degree of freedom belongs to at most two elements. This results in a cheap local communication when the method is parallelized on MIMD-machines. In addition, these two advantages of nonconforming finite element discretizations have to be combined 
with a fast solver in order to obtain an efficient computational method. Multigrid methods for nonconforming finite element approximations have been studied in a number of papers, e.g. see [4-11, 19, 21]. The first convergence proofs have been given by Braess/Verfürth [7] and Brenner [8] for the Poisson equation discretized by nonconforming piecewise linear elements. Later, these techniques have been extended to the Stokes equation in $[9,21]$ discretized by the nonconforming $P_{1} / P_{0^{-}}$ finite element and the $Q_{1}^{r o t} / Q_{0}$-finite element, respectively. In these papers, smoothers of Jacobi type and grid transfer operators have been used, which map discretely divergence free functions on the coarser level to discretely divergence free functions on the finer level. As a result, the convergence of the 2-level method for a sufficiently large number of smoothing steps could be established. The convergence rate behaves like $O\left(1 / \mathrm{m}^{1 / 4}\right)$ with respect to the number $m$ of smoothing steps. Replacing the nonconforming $P_{1}$ coarse grid correction by a conforming $P_{1}$ coarse grid correction, also the convergence of the $V$-cycle has been shown [10, 11]. In [6, 19], a new smoothing iteration has been proposed and studied in detail for the conforming Taylor-Hood element. This new method results in a convergence rate of $O(1 / m)$ with respect to the number $m$ of smoothing steps. Here, we will study, theoretically and numerically, this new type of smoothing iteration for nonconforming finite element discretizations of lowest order for the Stokes equation.

The paper is organized as follows. We formulate the continuous problem and describe its discretization by nonconforming elements of lowest order in Section 2. Then, in Section 3, we first explain the smoothing procedure of Braess-Sarazin type, afterwards the two-level method is defined and last special prolongation operators are studied. Finally, numerical experiments confirm the theoretical predictions in Section 4.

\section{The Problem and its Discretization}

\subsection{The Continuous Problem}

Let $\Omega \subset \mathbb{R}^{2}$ be a bounded, polygonal domain with boundary $\Gamma$. For any open subset $\omega$ of $\Omega$, we denote by $L^{2}(\omega)$ and $H^{k}(\omega), k \geq 1$, the standard Lebesgue and Sobolev spaces equipped with the norms $\|\cdot\|_{0, \omega}:=\|\cdot\|_{L^{2}(\omega)}$ and $\|\cdot\|_{k, \omega}:=\|\cdot\|_{H^{k}(\omega)}$ and with the inner product $(\cdot, \cdot)_{\omega}:=(\cdot, \cdot)_{L^{2}(\omega)}(\operatorname{cf}$. [1]). Since no confusion can arise, we use the same notations on the product spaces $L^{2}(\omega)^{2}$ and $H^{k}(\omega)^{2}$, respectively. We also make use of the seminorm $|\cdot|_{k, \omega}:=|\cdot|_{H^{k}(\omega)}$ in $H^{k}(\omega)$. If $\omega=\Omega$, we will omit the index $\omega$. Put

$$
\begin{aligned}
& V:=H_{0}^{1}(\Omega)^{2}:=\left\{\mathbf{v} \in H^{1}(\Omega)^{2}: \mathbf{v}=\mathbf{0} \text { on } \Gamma\right\}, \\
& Q:=L_{0}^{2}(\Omega):=\left\{q \in L^{2}(\Omega):(q, 1)=0\right\} .
\end{aligned}
$$

We consider the Stokes equation

$$
\begin{aligned}
&-\Delta \mathbf{u}+\nabla p=\mathbf{f} \text { in } \quad \Omega, \\
& \nabla \cdot \mathbf{u}=0 \quad \text { in } \Omega, \\
& \mathbf{u}=\mathbf{0} \quad \text { on } \Gamma,
\end{aligned}
$$


where $\mathbf{f} \in L^{2}(\Omega)^{2}$. The corresponding weak formulation reads:

Find $(\mathbf{u}, p) \in V \times Q$ such that for all $(\mathbf{v}, q) \in V \times Q$

$$
a(\mathbf{u}, \mathbf{v})+b(\mathbf{v}, p)+b(\mathbf{u}, q)=(\mathbf{f}, \mathbf{v}),
$$

where

$$
a(\mathbf{u}, \mathbf{v})=(\nabla \mathbf{u}, \nabla \mathbf{v}) \quad \text { and } \quad b(\mathbf{u}, q)=-(\nabla \cdot \mathbf{u}, q) .
$$

It is well known that (2) admits a unique solution.

\subsection{Nonconforming Finite Element Discretizations}

In this paper, we study nonconforming finite element discretizations of lowest order introduced by Crouzeix and Raviart for triangular meshes [12] and by Rannacher and Turek for quadrilateral meshes [17].

Let the coarse mesh $\mathscr{T}^{0}$ be a conforming decomposition of the domain $\Omega$ into elements $K \in \mathscr{T}^{0}$ which are allowed to be (open) triangles or convex quadrilaterals. Then, for a given mesh $\mathscr{T}^{l}$, we construct the next finer mesh $\mathscr{T}^{l+1}$ by subdividing each element into four "child elements". For that purpose, we connect the midpoints of the edges in the triangular case and the midpoints of opposite edges in the quadrilateral case. At the end of this refinement process, we get the finest level $l_{\max }$ and a final mesh $\mathscr{T}_{h}=\mathscr{T}^{l_{\max }}$.

On each triangulation $\mathscr{T}^{l}, l=0, \ldots, l_{\max }$, we construct finite element spaces $V^{l}, Q^{l}$ in the following way. Let $\mathscr{E}(K)$ denote the set of all edges of an element $K \in \mathscr{T}^{l}$, $\mathscr{E}^{\mathscr{l}}=\cup_{K} \mathscr{E}(K)$ the set of all edges of $\mathscr{T}^{l}, \mathscr{E}_{\Gamma}^{l}=\left\{E \in \mathscr{E}^{\mathscr{l}}: E \subset \Gamma\right\}$, and $\mathscr{E}_{0}^{l}=\mathscr{E}^{l} \backslash \mathscr{E}_{\Gamma} l^{\prime}$ the set of boundary and inner edges, respectively. For a piecewise continuous function $w$, the jump $[w]_{E}$ across an edge $E \in \mathscr{E} l$ is defined by

$$
[w]_{E}:= \begin{cases}\lim _{t \rightarrow+0}\left\{w\left(x+t \mathbf{n}_{E}\right)-w\left(x-t \mathbf{n}_{E}\right)\right\} & E \in \mathscr{E}_{0}^{l}, \\ \lim _{t \rightarrow+0}\left\{-w\left(x-t \mathbf{n}_{E}\right)\right\} & E \in \mathscr{E}_{\Gamma}^{l},\end{cases}
$$

where $\mathbf{n}_{E}$ is the normal unit vector on $E$ and $x \in E$. If $E \subset \Gamma$, we choose the orientation of $\mathbf{n}_{E}$ to be outward with respect to $\Omega$, otherwise $\mathbf{n}_{E}$ has an arbitrary but fixed orientation. The continuity condition of conforming finite elements at the edges $E \in \mathscr{E}^{l}$ is weakened to

$$
J_{E}\left(\mathbf{v}_{l}\right):=\frac{1}{|E|} \int_{E}\left[\mathbf{v}_{l}\right]_{E} d s=0 \quad \forall E \in \mathscr{E}^{l}, \quad \forall \mathbf{v}_{l} \in V^{l},
$$

where $|E|$ denotes the length of edge $E$.

We consider two types of nonconforming finite element spaces to approximate the velocity space $V$, in correspondence with the triangulation consisting of triangles or quadrilaterals. In fact, let 


$$
V^{l}:=\left\{\mathbf{v}_{l} \in L^{2}(\Omega)^{2}:\left.\mathbf{v}_{l}\right|_{K} \in P(K)^{2} \forall K \in \mathscr{T}^{l}, J_{E}\left(\mathbf{v}_{l}\right)=0 \forall E \in \mathscr{E}^{l}\right\},
$$

where $P(K):=P_{1}(K)$ if $K$ is a triangle and $P(K):=Q_{1}^{r o t}(K)$ for a quadrilateral element $K$. Here, $P_{1}(K)$ denotes the space of all linear functions on $K$ and $Q_{1}^{r o t}(K)$ the space of so-called "rotated bilinear" functions defined by

$$
Q_{1}^{r o t}(K):=\left\{\hat{q} \circ F_{K}^{-1}: \hat{q}=\operatorname{span}\left(1, \hat{x}_{1}, \hat{x}_{2}, \hat{x}_{1}^{2}-\hat{x}_{2}^{2}\right)\right\},
$$

where $F_{K}: \hat{K} \rightarrow K$ is the bilinear transformation between the reference element $\hat{K}=[-1,1]^{2}$ and the element $K$, see $[17,20]$. The degrees of freedom of a function $\mathbf{v}_{l} \in V^{l}$ are the mean values $N_{E}\left(\mathbf{v}_{l}\right)$ at the inner edges $E \in \mathscr{E}_{0}^{l}$ given by

$$
N_{E}\left(\mathbf{v}_{l}\right):=\left.\frac{1}{|E|} \int_{E} \mathbf{v}_{l}\right|_{K} d s \text { for } E \in \mathscr{E}(K),
$$

where $K$ is an arbitrary element having the edge $E$. Because of (3), the value $N_{E}\left(\mathbf{v}_{l}\right)$ is independent of the choice of $K$. Thus, the nodal values (4) are well defined for all $\mathbf{v} \in V+V^{l}$.

For approximating the pressure, we use piecewise constant functions, i.e.,

$$
Q^{l}:=\left\{q_{l} \in L^{2}(\Omega):\left.q_{l}\right|_{K} \in P_{0}(K) \quad \forall K \in \mathscr{T}^{l}\right\} \cap L_{0}^{2}(\Omega) .
$$

Now, on each level $l$, we consider the discrete problem.

Find $\left(\mathbf{u}_{l}, p_{l}\right) \in V^{l} \times Q^{l}$ such that for all $\left(\mathbf{v}_{l}, q_{l}\right) \in V^{l} \times Q^{l}$

$$
a_{l}\left(\mathbf{u}_{l}, \mathbf{v}_{l}\right)+b_{l}\left(\mathbf{v}_{l}, p_{l}\right)+b_{l}\left(\mathbf{u}_{l}, q_{l}\right)=\left(\mathbf{f}, \mathbf{v}_{l}\right),
$$

where the mesh dependent bilinear forms $a_{l}: V^{l} \times V^{l} \rightarrow \mathbb{R}, b_{l}: V^{l} \times Q^{l} \rightarrow \mathbb{R}$ are given by

$$
a_{l}\left(\mathbf{u}_{l}, \mathbf{v}_{l}\right)=\sum_{K \in \mathscr{T}^{l}}\left(\nabla \mathbf{u}_{l}, \nabla \mathbf{v}_{l}\right)_{K} \quad \text { and } \quad b_{l}\left(\mathbf{u}_{l}, q_{l}\right)=-\sum_{K \in \mathscr{T}^{l}}\left(\nabla \cdot \mathbf{u}_{l}, q_{l}\right)_{K} .
$$

It is well known that (5) is solvable and that the error estimate

$$
\left|\mathbf{u}-\mathbf{u}_{l}\right|_{1, h}+\left\|p-p_{l}\right\|_{0} \leq \operatorname{ch}_{l}\left(|\mathbf{u}|_{2}+|p|_{1}\right)
$$

holds, $[12,17,20]$. Note that $h_{l}$ is the mesh size parameter of the decomposition $\mathscr{T}^{l}$ and $|\cdot|_{1, h}$ is the discrete $H^{1}$-seminorm

$$
|v|_{1, h}:=\left(\sum_{K \in \mathscr{T}^{\prime \max }}|v|_{1, K}^{2}\right)^{1 / 2} .
$$

Under the additional assumption that $\Omega$ is convex, we have (cf. $[12,17]$ )

$$
\left\|\mathbf{u}-\mathbf{u}_{l}\right\|_{0} \leq \operatorname{ch}_{l}^{2}\left(|\mathbf{u}|_{2}+|p|_{1}\right) .
$$




\section{The Convergence of the 2-Level Method}

On each level $l$, the discrete problem (5) corresponds to a linear system of equations of the form

$$
\left(\begin{array}{cc}
A & B \\
B^{T} & 0
\end{array}\right)\left(\begin{array}{l}
U \\
P
\end{array}\right)=\left(\begin{array}{l}
F \\
0
\end{array}\right)
$$

where the dimension of the vectors coincides with the dimension of the finite element spaces $V^{l}$ and $Q^{l}$, respectively. Note that $A$ is a symmetric, positive definite matrix. To simplify the notation, the index $l$ will be omitted as long as no confusion can arise.

For the smoothing procedure on each level $l$, we will use the following iteration

$$
\left(\begin{array}{cc}
C & B \\
B^{T} & 0
\end{array}\right)\left(\begin{array}{c}
U^{j+1}-U^{j} \\
P^{j+1}-P^{j}
\end{array}\right)=\left(\begin{array}{c}
F \\
0
\end{array}\right)-\left(\begin{array}{cc}
A & B \\
B^{T} & 0
\end{array}\right)\left(\begin{array}{c}
U^{j} \\
P^{j}
\end{array}\right)
$$

which has been proposed in $[2,6,19]$. Here, we assume that the matrix $C$ has been generated by a continuous, elliptic and symmetric bilinear form $c:\left(V+V^{l}\right) \times\left(V+V^{l}\right) \rightarrow \mathbb{R}$.

The error for the next iterate only depends on the error of the velocity component of the previous iterate and is independent of the error of the pressure component. Moreover, for an arbitrary initial guess $\left(U^{0}, P^{0}\right)^{T}$, the iterates are discretely divergence free after one smoothing step, i.e.

$$
B^{T} U^{j}=0, \quad j \geq 1 .
$$

\subsection{The 2-Level Algorithm}

We shortly describe the 2-level method on the level $l, 1 \leq l \leq l_{\max }$ using $m_{1}$ presmoothing steps and $m_{2}$ post-smoothing steps. Because $Q^{l-1} \subset Q^{l}$, we can choose the natural injection as a prolongation of the discrete pressure. The prolongation of the discrete velocity will be denoted by $I_{l-1}^{l}: V^{l-1} \rightarrow V^{l}$.

Let $\left(\mathbf{u}_{l}^{0}, p_{l}^{0}\right)$ be a given approximation of the solution $\left(\mathbf{u}_{l}, p_{l}\right)$ on level $l$. For $n=0,1, \ldots$ define $\left(\mathbf{u}_{l}^{n, 0}, p_{l}^{n, 0}\right)=\left(\mathbf{u}_{l}^{n}, p_{l}^{n}\right)$ and perform

Step 1. Pre-smoothing: Solve for $j=0,1, \ldots, m_{1}-1$

$$
\begin{gathered}
c_{l}\left(\mathbf{u}_{l}^{n, j+1}-\mathbf{u}_{l}^{n, j}, \mathbf{v}_{l}\right)+b_{l}\left(\mathbf{v}_{l}, p_{l}^{n, j+1}-p_{l}^{n, j}\right) \\
=\left(\mathbf{f}, \mathbf{v}_{l}\right)-a_{l}\left(\mathbf{u}_{l}^{n, j}, \mathbf{v}_{l}\right)-b_{l}\left(\mathbf{v}_{l}, p_{l}^{n, j}\right) \quad \forall \mathbf{v}_{l} \in V^{l} \\
b_{l}\left(\mathbf{u}_{l}^{n, j+1}-\mathbf{u}_{l}^{n, j}, q_{l}\right)=-b_{l}\left(\mathbf{u}_{l}^{n, j}, q_{l}\right) \quad \forall q_{l} \in Q^{l} .
\end{gathered}
$$

Step 2. Coarse grid correction: Solve 


$$
\begin{aligned}
a_{l-1}\left(\mathbf{u}_{l-1}^{*}, \mathbf{v}_{l-1}\right)+ & b_{l-1}\left(\mathbf{v}_{l-1}, p_{l-1}^{*}\right) \\
= & \left(\mathbf{f}, I_{l-1}^{l} \mathbf{v}_{l-1}\right)-a_{l}\left(\mathbf{u}_{l}^{n, m_{1}}, I_{l-1}^{l} \mathbf{v}_{l-1}\right) \\
& -b_{l}\left(I_{l-1}^{l} \mathbf{v}_{l-1}, p_{l}^{n, m_{1}}\right) \quad \forall \mathbf{v}_{l-1} \in V^{l-1} \\
b_{l-1}\left(\mathbf{u}_{l-1}^{*}, q_{l-1}\right)= & 0 \quad \forall q_{l-1} \in Q^{l-1} .
\end{aligned}
$$

Define $\mathbf{u}_{l}^{n, m_{1}+1}:=\mathbf{u}_{l}^{n, m_{1}}+I_{l-1}^{l} \mathbf{u}_{l-1}^{*}$ and $p_{l}^{n, m_{1}+1}:=p_{l}^{n, m_{1}}+p_{l-1}^{*}$.

Step 3. Post-smoothing: Solve for $j=0,1, \ldots, m_{2}-1$

$$
\begin{aligned}
& c_{l}\left(\mathbf{u}_{l}^{n, m_{1}+j+2}-\mathbf{u}_{l}^{n, m_{1}+j+1}, \mathbf{v}_{l}\right)+b_{l}\left(\mathbf{v}_{l}, p_{l}^{n, m_{1}+j+2}-p_{l}^{n, m_{1}+j+1}\right) \\
& =\left(\mathbf{f}, \mathbf{v}_{l}\right)-a_{l}\left(\mathbf{u}_{l}^{n, m_{1}+j+1}, \mathbf{v}_{l}\right)-b_{l}\left(\mathbf{v}_{l}, p_{l}^{n, m_{1}+j+1}\right) \quad \forall \mathbf{v}_{l} \in V^{l} \\
& b_{l}\left(\mathbf{u}_{l}^{n, m_{1}+j+2}-\mathbf{u}_{l}^{n, m_{1}+j+1}, q_{l}\right)=-b_{l}\left(\mathbf{u}_{l}^{n, m_{1}+j+1}, q_{l}\right) \quad \forall q_{l} \in Q^{l} .
\end{aligned}
$$

Choose $\mathbf{u}_{l}^{n+1}:=\mathbf{u}_{l}^{n, m_{1}+m_{2}+1}, p_{l}^{n+1}:=p_{l}^{n, m_{1}+m_{2}+1}$.

In the following, we will show that the two-level method converges for sufficiently many smoothing steps. As a consequence, the $\mathrm{W}$-cycle multigrid algorithm is convergent, too (cf. [3, 13]).

We follow the general framework of proving a smoothing property and an approximation property. Putting both properties together, the convergence of the 2-level method is obtained.

\subsection{The Smoothing Property}

In the following, we denote by $A_{l}, B_{l}, C_{l}$ the matrices $A, B, C$ in (7) and (8) on level $l$ and by $I_{l}$ the identity with $\operatorname{dim} I_{l}=\operatorname{dim} A_{l}$. For simplicity, we consider the case when $C_{l}=\alpha_{l} I_{l}$ and the parameter $\alpha_{l}$ satisfies $\alpha_{l} \geq \lambda_{\max }\left(A_{l}\right)$, where $\lambda_{\max }\left(A_{l}\right)$ is the largest eigenvalue of $A_{l}$. Note that the size of $\lambda_{\max }\left(A_{l}\right)$ depends on the basis functions used to span $V^{l}$. In contrast to [6], we take the standard basis $\left\{\Phi_{i}\right\}$ such that the mean value $N_{E}\left(\Phi_{i}\right)$ is equal to $(0,0),(1,0)$ or $(0,1)$. As a consequence, $\lambda_{\max }\left(A_{l}\right)$ is uniformly bounded by a level-independent constant. Let the discrete $l_{2}$ norm of a vector $U=\left(U_{i}\right)$ be denoted by

$$
\|U\|_{0, d}:=\left(\sum_{i} U_{i}^{2}\right)^{1 / 2}
$$

This choice is connected with the norm equivalence on each level $l, 0 \leq l \leq l_{\max }$,

$$
\frac{c_{1}}{h_{l}}\left\|\mathbf{u}_{l}\right\|_{0} \leq\|U\|_{0, d} \leq \frac{c_{2}}{h_{l}}\left\|\mathbf{u}_{l}\right\|_{0}, \quad \quad \mathbf{u}_{l}=\sum_{i} U_{i} \Phi_{i} .
$$

Lemma 1. On level $l$, let $(U, P)$ be the solution of the discrete problem $(7),\left(U^{m}, P^{m}\right)$ be the final iterate after $m \geq 2$ smoothing steps of the relaxation (8) with the initial guess $\left(U^{0}, P^{0}\right)$. Then, there is a level independent constant $c$ such that 


$$
\left\|A_{l}\left(U^{m}-U\right)+B_{l}\left(P^{m}-P\right)\right\|_{0, d} \leq \frac{c}{m}\left\|U^{0}-U\right\|_{0, d}
$$

holds.

Proof: The proof is based on purely algebraic properties and has been given in [4].

\section{3. $L^{2}$-Orthogonal Prolongations}

We consider the case of $L^{2}$-orthogonal prolongations, i.e., $I_{l-1}^{l}: V+V^{l-1} \rightarrow V^{l}$ is given by

$$
\left(I_{l-1}^{l} \mathbf{v}-\mathbf{v}, \mathbf{w}_{l}\right)=0 \quad \forall \mathbf{w}_{l} \in V^{l}, \quad \mathbf{v} \in V+V^{l-1} .
$$

Then, we can show the approximation property based on ideas developed in [7] for the $P_{1}$-nonconforming finite element discretization applied to the Poisson equation. Let $\left(\mathbf{u}_{l}^{m}, p_{l}^{m}\right) \in V^{l} \times Q^{l}$ be the approximate solution after $m$ smoothing steps and $\left(\mathbf{u}_{l-1}^{*}, p_{l-1}^{*}\right)$ the coarse grid correction defined in (9).

Define the auxiliary variational problem

$$
\begin{aligned}
a(\mathbf{z}, \mathbf{v})+b(\mathbf{v}, \phi) & =(\mathbf{g}, \mathbf{v}) \quad \forall \mathbf{v} \in V, \\
b(\mathbf{z}, \psi) & =0 \quad \forall \psi \in Q,
\end{aligned}
$$

where $\mathbf{g} \in V^{l} \subset L^{2}(\Omega)^{2}$ denotes the Riesz-Fischer representation of the defect $F_{l}-A_{l} U^{m}-B_{l} P^{m}$ given by

$$
\left(\mathbf{g}, \mathbf{w}_{l}\right)=\left(\mathbf{f}, \mathbf{w}_{l}\right)-a_{l}\left(\mathbf{u}_{l}^{m}, \mathbf{w}_{l}\right)-b_{l}\left(\mathbf{w}_{l}, p_{l}^{m}\right) \quad \forall \mathbf{w}_{l} \in V^{l} .
$$

Under the assumption that the solution of (11) is $H^{2} \times H^{1}$-regular, we have

$$
\|\mathbf{z}\|_{2}+\|\phi\|_{1} \leq c\|\mathbf{g}\|_{0}
$$

Setting $\mathbf{w}_{l}=I_{l-1}^{l} \mathbf{v}_{l-1}$ in (12) and using the $L^{2}$-orthogonality of the prolongation, we see that the coarse grid correction is the nonconforming finite element approximation of (11)

$$
\begin{gathered}
a_{l-1}\left(\mathbf{u}_{l-1}^{*}, \mathbf{v}_{l-1}\right)+b_{l-1}\left(\mathbf{v}_{l-1}, p_{l-1}^{*}\right)=\left(\mathbf{g}, \mathbf{v}_{l-1}\right) \quad \forall \mathbf{v}_{l-1} \in V^{l-1}, \\
b_{l-1}\left(\mathbf{u}_{l-1}^{*}, q_{l-1}\right)=0 \quad \forall q_{l-1} \in Q^{l-1} .
\end{gathered}
$$

On the other hand, $\left(\mathbf{u}_{l}-\mathbf{u}_{l}^{m}, p_{l}-p_{l}^{m}\right)$ is the nonconforming finite element approximation of $(\mathbf{z}, \phi)$ on the level $l$. Indeed, using (5), (12), and that $\mathbf{u}_{l}^{m}$ is discretely divergence free, we have

$$
\begin{aligned}
a_{l}\left(\mathbf{u}_{l}-\mathbf{u}_{l}^{m}, \mathbf{w}_{l}\right)+b_{l}\left(\mathbf{w}_{l}, p_{l}-p_{l}^{m}\right) & =\left(\mathbf{g}, \mathbf{w}_{l}\right) \quad \forall \mathbf{w}_{l} \in V^{l}, \\
b_{l}\left(\mathbf{u}_{l}-\mathbf{u}_{l}^{m}, q_{l}\right) & =0 \quad \forall q_{l} \in Q^{l} .
\end{aligned}
$$


Consequently, the $L^{2}$-stability of the prolongation

$$
\left\|I_{l-1}^{l} \mathbf{v}\right\|_{0} \leq\|\mathbf{v}\|_{0} \quad \forall \mathbf{v} \in V+V^{l-1},
$$

the error estimate (6) on the level $l$ and $l-1$ and the stability result (13) imply

$$
\begin{aligned}
\left\|\mathbf{u}_{l}-\mathbf{u}_{l}^{m}-I_{l-1}^{l} \mathbf{u}_{l-1}^{*}\right\|_{0} & =\left\|I_{l-1}^{l}\left(\mathbf{u}_{l}-\mathbf{u}_{l}^{m}-\mathbf{u}_{l-1}^{*}\right)\right\|_{0} \\
& \leq\left\|\mathbf{u}_{l}-\mathbf{u}_{l}^{m}-\mathbf{z}\right\|_{0}+\left\|\mathbf{z}-\mathbf{u}_{l-1}^{*}\right\|_{0} \\
& \leq c\left(h_{l}^{2}+h_{l-1}^{2}\right)\|\mathbf{g}\|_{0} .
\end{aligned}
$$

Now, we estimate the $L^{2}$-norm of $g$ by the discrete $l_{2}$-norm of the defect $A_{l}\left(U-U^{m}\right)+B_{l}\left(P-P^{m}\right)$. Assuming that the representation of $\mathbf{g} \in V^{l}$ is given by

$$
\mathbf{g}=\sum_{i} g_{i} \Phi_{i}
$$

we have

$$
\|\mathbf{g}\|_{0}^{2}=\sum_{i} g_{i}\left(\mathbf{g}, \Phi_{i}\right) \leq\left(\sum_{i} g_{i}^{2}\right)^{1 / 2}\left(\sum_{i}\left(\mathbf{g}, \Phi_{i}\right)^{2}\right)^{1 / 2} .
$$

On level $l$, the discrete $l_{2}$-norm can be estimated by the $L^{2}$-norm as follows

$$
\left(\sum_{i} g_{i}^{2}\right)^{1 / 2}=\|g\|_{0, d} \leq \frac{c}{h_{l}}\|\mathbf{g}\|_{0} .
$$

Taking into consideration that

$$
\left\|A_{l}\left(U-U^{m}\right)+B_{l}\left(P-P^{m}\right)\right\|_{0, d}=\left(\sum_{i}\left(\mathbf{g}, \Phi_{i}\right)^{2}\right)^{1 / 2},
$$

we finally get

$$
\|\mathbf{g}\|_{0} \leq \frac{c}{h_{l}}\left\|A_{l}\left(U-U^{m}\right)+B_{l}\left(P-P^{m}\right)\right\|_{0, d}
$$

which, with (14), proves the following approximation property.

Lemma 2. Let $\left(\mathbf{u}_{l}, p_{l}\right)$ be the solution of $(5),\left(\mathbf{u}_{l}^{m}, p_{l}^{m}\right)$ be the approximate solution after $m$ smoothing steps and $\left(\mathbf{u}_{l-1}^{*}, p_{l-1}^{*}\right)$ the coarse grid correction. Then, we have

$$
\left\|\mathbf{u}_{l}-\mathbf{u}_{l}^{m}-I_{l-1}^{l} \mathbf{u}_{l-1}^{*}\right\|_{0} \leq c h_{l}\left\|A_{l}\left(U-U^{m}\right)+B_{l}\left(P-P^{m}\right)\right\|_{0, d} .
$$

From the smoothing and approximation property, we obtain 
Theorem 3. Let the solution of (11) be $H^{2} \times H^{1}$-regular and $C_{l}=\alpha_{l} I_{l}$ with $\alpha_{l} \geq \lambda_{\max }\left(A_{l}\right)$. Then, the 2-level method convergences in the $L^{2}$-norm of the velocity for a $L^{2}$-orthogonal prolongation operator $I_{l-1}^{l}$ and sufficiently many smoothing steps $m \geq 2$. In particular, there is a level independent constant $c$ such that

$$
\left\|\mathbf{u}_{l}-\mathbf{u}_{l}^{m}-I_{l-1}^{l} \mathbf{u}_{l-1}^{*}\right\|_{0} \leq \frac{c}{m}\left\|\mathbf{u}_{l}-\mathbf{u}_{l}^{0}\right\|_{0}
$$

or equivalently

$$
\left\|U-U_{l}^{m}-U_{l}^{m+1}\right\|_{0, d} \leq \frac{c}{m}\left\|U-U^{0}\right\|_{0, d},
$$

where $U_{l}^{m+1}$ is the corresponding vector representation of $I_{l-1}^{l} \mathbf{u}_{l-1}^{*}$.

Proof: Starting with the approximation property, using the smoothing property and the norm equivalence, we get

$$
\begin{aligned}
\left\|\mathbf{u}_{l}-\mathbf{u}_{l}^{m}-I_{l-1}^{l} \mathbf{u}_{l-1}^{*}\right\|_{0} & \leq c h_{l}\left\|A_{l}\left(U-U^{m}\right)+B_{l}\left(P-P^{m}\right)\right\|_{0, d} \\
& \leq \frac{c h_{l}}{m}\left\|U-U^{0}\right\|_{0, d} \\
& \leq \frac{c}{m}\left\|\mathbf{u}_{l}-\mathbf{u}_{l}^{0}\right\|_{0} .
\end{aligned}
$$

Owing to the norm equivalence, we conclude (16).

\section{4. $L^{2}$-Bounded Prolongations}

Note that the use of $L^{2}$-prolongations in the $Q_{1}^{\text {rot }} / Q_{0}$-case is too expensive since the standard basis functions are not $L^{2}$-orthogonal as it is the case of the $P_{1}$-nonconforming finite element. Hence, the possibility to use an $L^{2}$-bounded prolongation would simplify the multigrid method considerably.

A careful inspection of the convergence proof for the 2-level cycle shows that the prolongations have not to be $L^{2}$-orthogonal. Indeed, in (14), we used only the $L^{2}$-boundedness of the prolongation $I_{l-1}^{l}$. The second idea of the proof, to interpret $\mathbf{u}_{l}-\mathbf{u}_{l}^{m}$ and $\mathbf{u}_{l-1}^{*}$ as nonconforming finite element discretizations of an appropriate auxiliary problem, can be also conserved by a clever definition of the Riesz-Fischer representation of the defect. This has been focussed in a recent paper [5] in a general setting to handle the mortar finite element method. Here, we explain the technique for the nonconforming $P_{1} / P_{0}$ and the nonconforming $Q_{1}^{r o t} / Q_{0}$-finite element method.

Let $W^{l}$ denote the space given by

$$
W^{l}:=\left\{\mathbf{w}_{l} \in L^{2}(\Omega)^{2}:\left.\mathbf{w}_{l}\right|_{K} \in P(K)^{2} \quad \forall K \in \mathscr{T}^{l}\right\},
$$

with $P(K):=P_{1}(K)$ and $P(K):=Q_{1}^{r o t}(K)$, respectively. We have no additional assumptions to guarantee any weak continuity conditions at the edges $E \in \mathscr{E} l$. A 
basis of $W^{l}$ consists of functions $\tilde{\Phi}_{i}$ such that the (one-sided) mean values $N_{E}\left(\left.\tilde{\Phi}_{i}\right|_{K}\right)$ are $(0,0),(1,0)$ or $(0,1)$. Again, the norm equivalence

$$
\frac{c_{3}}{h_{l}}\left\|\mathbf{w}_{l}\right\|_{0} \leq\|W\|_{0, d} \leq \frac{c_{4}}{h_{l}}\left\|\mathbf{w}_{l}\right\|_{0}, \quad w_{l}=\sum_{i} W_{i} \tilde{\Phi}_{i},
$$

between the $L^{2}$-norm of a function in $W^{l}$ and the discrete $l_{2}$-norm of its vector representation is satisfied. Moreover, we see that $V^{l-1}, V^{l}$ are contained in $W^{l}$.

Let us define the prolongation $I_{l-1}^{l}: V+W^{l} \rightarrow V^{l}$ by a simple averaging over the edges such that

$$
N_{E}\left(I_{l-1}^{l} \mathbf{w}\right):=\frac{1}{2|E|} \int_{E}\left(\left.\mathbf{w}\right|_{K_{1}}+\left.\mathbf{w}\right|_{K_{2}}\right) d s \quad \forall E=\partial K_{1} \cap \partial K_{2} \in \mathscr{E} l .
$$

The properties of this prolongation are summarized in the next lemma.

Lemma 4. The prolongation $I_{l-1}^{l}$, defined in (17), satisfies

$$
\begin{aligned}
\left\|I_{l-1}^{l} \mathbf{w}\right\|_{0} & \leq c\|\mathbf{w}\|_{0}, \quad \forall \mathbf{w} \in W^{l}, \\
I_{l-1}^{l} \mathbf{w} & =\mathbf{w}, \quad \forall w \in V^{l} .
\end{aligned}
$$

Proof: The first statement follows from the definition of $I_{l-1}^{l}$ and the norm equivalence. The second one is a consequence of imposing the weak continuity condition over edges for elements of $V^{l}$.

Now, we can define the Riesz-Fischer representation $\mathbf{g} \in W^{l} \subset L^{2}(\Omega)^{2}$ of the defect by

$$
(\mathbf{g}, \mathbf{w})=\left(\mathbf{f}, I_{l-1}^{l} \mathbf{w}\right)-a_{l}\left(\mathbf{u}_{l}^{m}, I_{l-1}^{l} \mathbf{w}\right)-b_{l}\left(I_{l-1}^{l} \mathbf{w}, p_{l}^{m}\right) \quad \forall \mathbf{w} \in W^{l}
$$

and consider the auxiliary problem (11). Then, $\mathbf{u}_{l}-\mathbf{u}_{l}^{m}$ and $\mathbf{u}_{l-1}^{*}$ can be considered again as finite element approximations of the first component of the solution $(\mathbf{z}, \phi)$ of (11).

Repeating the arguments for proving Lemma 2, we obtain

Theorem 5. Let the solution of (11) be $H^{2} \times H^{1}$-regular and $C_{l}=\alpha_{l} I_{l}$ with $\alpha_{l} \geq \lambda_{\max }\left(A_{l}\right)$. Then, the 2-level method converges in the $L^{2}$-norm of the velocity for the prolongation operator $I_{l-1}^{l}$, defined by (17), and sufficiently many smoothing steps $m \geq 2$. In particular, we have

$$
\left\|\mathbf{u}_{l}-\mathbf{u}_{l}^{m}-I_{l-1}^{l} \mathbf{u}_{l-1}^{*}\right\|_{0} \leq \frac{c}{m}\left\|\mathbf{u}_{l}-\mathbf{u}_{l}^{0}\right\|_{0}
$$

or equivalently 


$$
\left\|U-U_{l}^{m}-U_{l}^{m+1}\right\|_{0, d} \leq \frac{c}{m}\left\|U-U^{0}\right\|_{0, d}
$$

where $U_{l}^{m+1}$ is the corresponding vector representation of $I_{l-1}^{l} \mathbf{u}_{l-1}^{*}$.

\section{Numerical Studies}

Numerical experiments have been carried out for the nonconforming $P_{1} / P_{0}$-finite element discretization [12] with the $L^{2}$-projection as prolongation operator $I_{l-1}^{l}$.

In order to perform one smoothing step, we have to solve the saddle point problem (8). For this, we apply a pressure Schur complement method, i.e. first we solve the Schur complement equation for the pressure

$$
B^{T} C^{-1} B\left(P^{j+1}-P^{j}\right)=B^{T} C^{-1}\left(F-A U^{j}-B P^{j}\right)+B^{T} U^{j}
$$

with an iterative scheme: second, the velocity component is computed by

$$
U^{j+1}-U^{j}=C^{-1}\left[\left(F-A U^{j}-B P^{j}\right)-B\left(P^{j+1}-P^{j}\right)\right] .
$$

Example 1. This example has been designed to confirm the theoretically predicted results with respect to the 2-level method. We consider the Stokes equation (1) in the unit square with $\mathbf{f}=\mathbf{0}$, such that $\mathbf{u}=\mathbf{0}, p=0$ is the solution of (1). The computations were carried out on a sequence of meshes starting with Grid 1 (Fig. 1). The discrete solution on each level is $U_{l}=0, P_{l}=0$.

As the initial guess of the 2-level method we have chosen $U_{l, i}=1$ for all interior degrees of freedom and $P_{l, i}=0$ on each level. Thus, the initial error is smooth. The number of pre-smoothing steps is denoted by $m$ and the number of postsmoothing steps was set to be zero. The approximation of $A_{l}^{-1}$ in the BraessSarazin-type smoother was chosen $\left(\alpha I_{l}\right)^{-1}$ with the damping parameter $\alpha_{l}=2 \max _{i}\left\{a_{i i}^{l}\right\}=16, l=0,1, \ldots$, where $a_{i i}^{l}$ is the $\mathrm{i}$-th diagonal entry of $A_{l}$. Owing
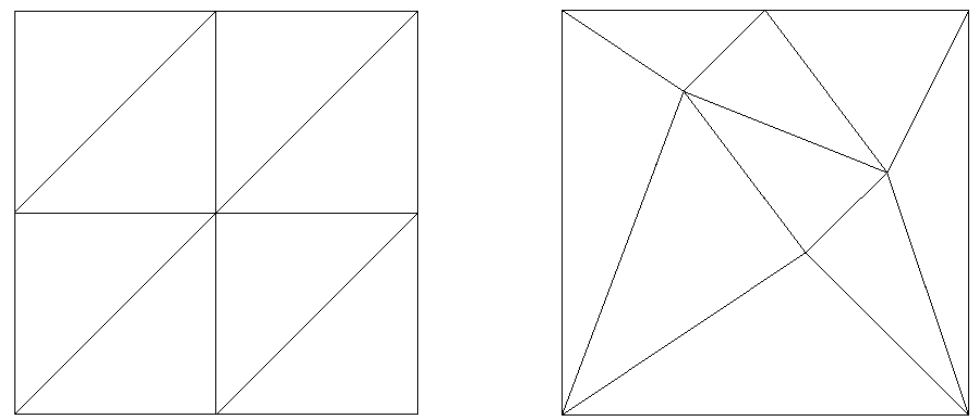

Figure 1. Grid 1 (left) and Grid 2 (right), level 0 
to the Geršgorin theorem, $\alpha_{l} \geq \lambda_{\max }\left(A_{l}\right)$. Thus, we have exactly the situation investigated in Section 3.

The results of the numerical studies are given in Tables 1 and 2. Table 1 shows the geometric mean of the smoothing rate

$$
\frac{\left\|A_{l} U^{m}-B_{l} P^{m}\right\|_{0, d}}{\left\|U^{0}\right\|_{0, d}},
$$

over several 2-level steps (cf. [10]), and Table 2 the geometric mean of the error reduction rate

$$
\frac{\left\|U_{l}^{m}+U_{l}^{m+1}\right\|_{0, d}}{\left\|U^{0}\right\|_{0, d}},
$$

over several 2-level steps (cf. [16]). The following observations can be made:

- Both rates are independent of the level.

- The rates decrease like $O(1 / m)$ with the number $m$ of smoothing steps, see also Fig. 2 for a graphical representation.

- In this example, the constant in the smoothing rate is approximately 38 and the constant in the error reduction rate is about 56 . This means that we need at least $m=6$ smoothing steps for the convergence of the 2-level method.

Example 2. This example presents numerical studies of multigrid methods with Braess-Sarazin-type smoothers. We have studied the same example as in [6] to compare our results with those of the conforming modified Taylor-Hood finite

Table 1. Average smoothing rate for the 2-level method, Example 1

\begin{tabular}{ccccc}
\hline$m \backslash$ level & 3 & 4 & 5 & 6 \\
\hline 6 & 0.641 & 0.624 & 0.605 & 0.601 \\
8 & 0.504 & 0.483 & 0.470 & 0.472 \\
10 & 0.409 & 0.386 & 0.380 & 0.381 \\
12 & 0.341 & 0.319 & 0.317 & 0.317 \\
16 & 0.253 & 0.237 & 0.238 & 0.238 \\
20 & 0.202 & 0.191 & 0.192 & 0.192 \\
24 & 0.168 & 0.160 & 0.161 & 0.161 \\
\hline
\end{tabular}

Table 2. Average error reduction rate for the 2-level method, Example 1

\begin{tabular}{ccccc}
\hline$m \backslash$ level & 3 & 4 & 5 & 6 \\
\hline 6 & 0.831 & 0.874 & 0.877 & 0.875 \\
8 & 0.667 & 0.696 & 0.695 & 0.693 \\
10 & 0.550 & 0.570 & 0.567 & 0.567 \\
12 & 0.463 & 0.477 & 0.475 & 0.475 \\
16 & 0.347 & 0.356 & 0.355 & 0.355 \\
20 & 0.274 & 0.281 & 0.281 & 0.281 \\
24 & 0.223 & 0.230 & 0.230 & 0.230 \\
\hline
\end{tabular}




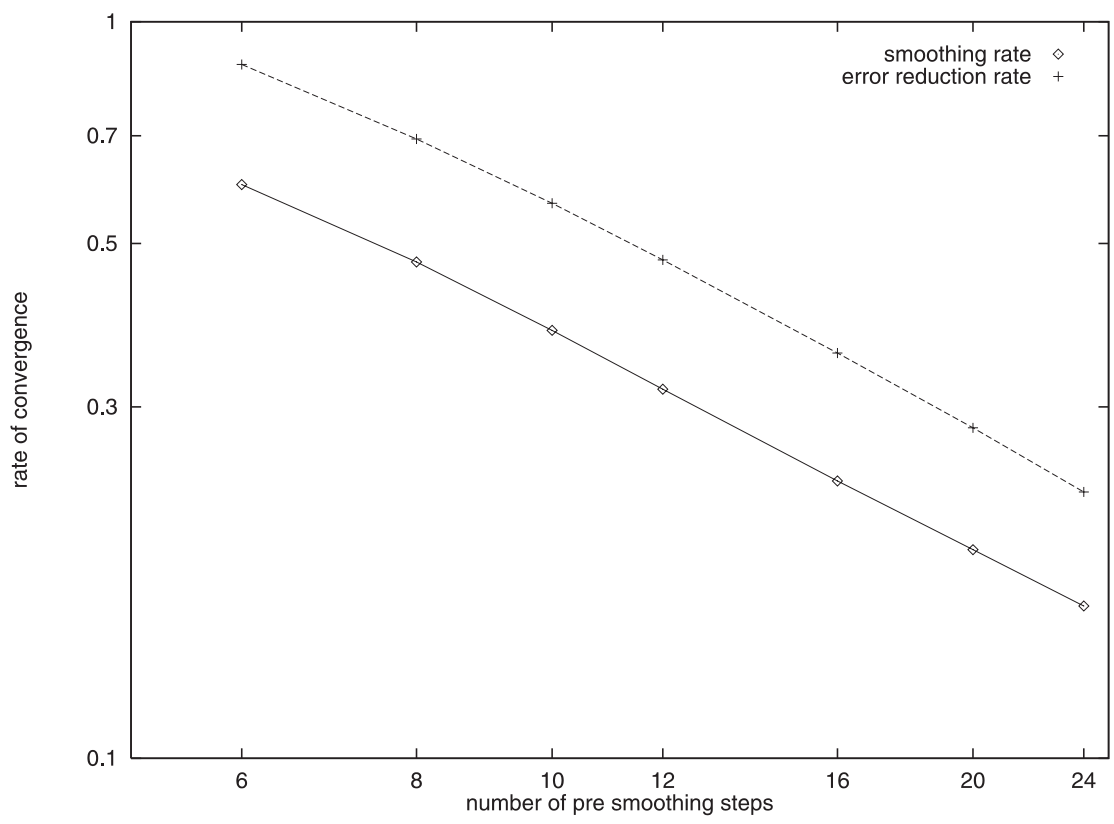

Figure 2. Rates of convergence for different numbers of smoothing steps, Example 1, level 6

element discretization. Thus, we consider the Stokes equations in the unit square where the right hand side and the boundary conditions are chosen such that

$$
\begin{aligned}
& \mathbf{u}=(\sin (x) \sin (y), \cos (x) \cos (y))^{T}, \\
& p=2 \cos (x) \sin (y)+\text { constant }
\end{aligned}
$$

is the solution. As approximations of $A^{-1}$ within the Braess-Sarazin-type smoother, we have studied the inverse of the diagonal of $A\left(C^{-1}=\operatorname{diag}(A)^{-1}\right)$, one step of a SSOR-iteration with the damping parameter $\omega=1\left(\mathrm{C}^{-1}=\operatorname{SSOR}(A)\right)$, and one step of a $\operatorname{ILU}_{\beta}$-iteration $\left(C^{-1}=I L U_{\beta}(A)\right)$, where $\beta$ is a diagonal compensation factor. In the ILU $\mathrm{U}_{\beta}$-iteration, the absolute value of each computed nonzero entry which does not belong to the sparsity pattern of $A$ is multiplied by $\beta$ and added to the diagonal of the upper part of the decomposition. The Schur complement systems (18) with $C^{-1}=\operatorname{diag}(A)^{-1}$ and $C^{-1}=\operatorname{SSOR}(A)$ have been solved approximately by the conjugate gradient method [14]. For the approximate solution of the systems with $C^{-1}=\operatorname{ILU}_{\beta}(A)$, we used gmres [18]. The pressure Schur complement iterations stopped either after having reduced the norm of the residual by the factor 0.1 or after 10 steps. In each test, the damping parameters $\alpha_{l}=\alpha$ have been fixed for all levels.

The results of the numerical tests are presented in Tables 3 and 4. The most important observations are the followings:

- The choice of $C^{-1}$ is essential for the behaviour of the multigrid method. In our tests, $C^{-1}=\operatorname{ILU}_{0.0}(A)$ was clearly the best choice. With this choice, we obtained acceptable rates of convergence for the $\mathrm{W}$-cycles on both grids. The choice $\beta=1.0$ of the diagonal compensation factor in the ILU-decomposition 
Table 3. Average error reduction rates, Example 2, Grid 1

\begin{tabular}{|c|c|c|c|c|c|c|}
\hline Method & $C^{-1}$ & $\alpha$ & 4 & 5 & 6 & 7 \\
\hline $\begin{array}{l}\mathrm{W}(3,3) \\
\mathrm{W}(3,3) \\
\mathrm{W}(3,3)\end{array}$ & $\begin{array}{l}\operatorname{diag}(A)^{-1} \\
\operatorname{diag}(A)^{-1} \\
\operatorname{diag}(A)^{-1}\end{array}$ & $\begin{array}{l}1.00 \\
1.25 \\
1.50\end{array}$ & $\begin{array}{l}0.57 \\
0.57 \\
0.70\end{array}$ & $\begin{array}{l}0.63 \\
0.90 \\
0.89\end{array}$ & $\begin{array}{l}0.58 \\
0.68 \\
0.93\end{array}$ & $\begin{array}{l}0.60 \\
0.70 \\
0.97\end{array}$ \\
\hline $\begin{array}{l}\mathrm{W}(2,2) \\
\mathrm{W}(3,3) \\
\mathrm{W}(4,4) \\
\mathrm{W}(5,5) \\
\mathrm{W}(6,6) \\
\mathrm{W}(2,2) \\
\mathrm{V}(4,4)\end{array}$ & $\begin{array}{l}\text { SSOR(A) } \\
\text { SSOR(A) } \\
\text { SSOR(A) } \\
\text { SSOR(A) } \\
\text { SSOR(A) } \\
\text { SSOR(A) } \\
\text { SSOR(A) }\end{array}$ & $\begin{array}{l}1.00 \\
1.00 \\
1.00 \\
1.00 \\
1.00 \\
1.50 \\
1.00\end{array}$ & $\begin{array}{l}0.56 \\
0.42 \\
0.34 \\
0.24 \\
0.19 \\
0.54 \\
0.27\end{array}$ & $\begin{array}{l}0.55 \\
0.43 \\
0.33 \\
0.25 \\
0.19 \\
0.67 \\
0.44\end{array}$ & $\begin{array}{l}0.56 \\
0.42 \\
0.33 \\
0.25 \\
0.18 \\
0.65 \\
0.55\end{array}$ & $\begin{array}{l}0.56 \\
0.42 \\
0.32 \\
0.24 \\
0.18 \\
0.64 \\
0.70\end{array}$ \\
\hline $\begin{array}{l}\mathrm{W}(2,2) \\
\mathrm{W}(2,2) \\
\mathrm{V}(2,2) \\
\mathrm{V}(2,2)\end{array}$ & $\begin{array}{l}\operatorname{ILU}_{0.0}(\mathrm{~A}) \\
\operatorname{ILU}_{1.0}(\mathrm{~A}) \\
\operatorname{ILU}_{0.0}(\mathrm{~A}) \\
\mathrm{ILU}_{1.0}(\mathrm{~A})\end{array}$ & $\begin{array}{l}1.00 \\
1.00 \\
1.00 \\
1.00\end{array}$ & $\begin{array}{l}0.17 \\
0.35 \\
0.16 \\
0.38\end{array}$ & $\begin{array}{l}0.14 \\
0.33 \\
0.17 \\
0.44\end{array}$ & $\begin{array}{l}0.19 \\
0.32 \\
0.32 \\
0.68\end{array}$ & $\begin{array}{l}0.18 \\
0.33 \\
0.44 \\
0.79\end{array}$ \\
\hline \multicolumn{3}{|c|}{$\begin{array}{l}\text { velocity degrees of freedom } \\
\text { pressure degrees of freedom }\end{array}$} & $\begin{array}{l}6016 \\
2048\end{array}$ & $\begin{array}{l}24320 \\
8192\end{array}$ & $\begin{array}{l}97792 \\
32768\end{array}$ & $\begin{array}{l}392192 \\
131072\end{array}$ \\
\hline
\end{tabular}

Table 4. Average error reduction rates, Example 2, Grid 2

\begin{tabular}{lllllll}
\hline Method & $C^{-1}$ & $\alpha$ & 4 & 5 & 6 & 7 \\
\hline $\mathrm{W}(3,3)$ & SSOR(A) & 1.0 & 0.69 & 0.67 & 0.66 & 0.66 \\
$\mathrm{~V}(10,10)$ & $\mathrm{SSOR}(\mathrm{A})$ & 1.0 & 0.34 & 0.84 & div. & \\
$\mathrm{W}(2,2)$ & $\mathrm{ILU}_{0.0}(\mathrm{~A})$ & 1.0 & 0.32 & 0.28 & 0.30 & 0.30 \\
$\mathrm{~W}(3,3)$ & $\mathrm{ILU}_{0.0}(\mathrm{~A})$ & 1.0 & 0.20 & 0.22 & 0.22 & 0.22 \\
$\mathrm{~W}(4,4)$ & $\mathrm{ILU}_{0.0}(\mathrm{~A})$ & 1.0 & 0.12 & 0.17 & 0.18 & 0.18 \\
$\mathrm{~W}(5,5)$ & $\mathrm{ILU}_{0.0}(\mathrm{~A})$ & 1.0 & 0.07 & 0.13 & 0.16 & 0.13 \\
$\mathrm{~W}(6,6)$ & $\mathrm{ILU}_{0.0}(\mathrm{~A})$ & 1.0 & 0.05 & 0.09 & 0.13 & 0.11 \\
$\mathrm{~W}(2,2)$ & $\mathrm{ILU}_{1.0}(\mathrm{~A})$ & 1.0 & 0.41 & 0.42 & 0.43 & 0.43 \\
$\mathrm{~V}(4,4)$ & $\mathrm{ILU}_{0.0}(\mathrm{~A})$ & 1.0 & 0.13 & 0.23 & 0.31 & 0.39 \\
$\mathrm{~V}(4,4)$ & $\mathrm{ILU}_{1.0}(\mathrm{~A})$ & 1.0 & 0.31 & 0.47 & 0.60 & 0.73 \\
velocity degrees of freedom & 6832 & 27488 & 110272 & 441728 \\
pressure degrees of freedom & 2304 & 9216 & 36864 & 147456 \\
\hline
\end{tabular}

worsened the convergence rates considerably. But these rates are still better than for $C^{-1}=\operatorname{SSOR}(A)$. The worst behaviour is observed for $C^{-1}=\operatorname{diag}(A)^{-1}$. With this choice, we were not able to solve the problem on Grid 2.

- In nearly all tests with the W-cycle, the independency of the rates of convergence of the level could be observed despite of solving the Schur complement system (18) within the Braess-Sarazin-type smoother only approximately. The improvement of the error reduction rates is approximately linear with the number of smoothing steps, see $\mathrm{W}(2,2)-\mathrm{W}(6,6)$-cycle for $C^{-1}=\operatorname{SSOR}(A)$ in Table 3 and for $C^{-1}=I L U_{0.0}(A)$ in Table 4.

- The rates of convergence of the $\mathrm{V}$-cycle increase in general with increasing level of refinement. Note that there is no theoretical convergence proof for the $\mathrm{V}$-cycle algorithm. Therefore, in a number of papers $[10,11]$ conforming coarse grid corrections have been studied.

- Unfortunately, the rates of convergence are not as good as the rates given in [6] for the conforming modified Taylor-Hood finite element discretization, e.g. for 
the $\mathrm{W}(3,3)$-cycle, $C^{-1}=\operatorname{SSOR}(A), \alpha=1$, Grid 2, the rates of convergence are 0.095 in [6, Table 3] and 0.66 here (level 7). Up to now, we have no theoretical explanation for this observation.

\title{
References
}

[1] Adams, R. A.: Sobolev spaces. New York: Academic Press, 1975.

[2] Bank, R. E., Welfert, B. D., Yserentant, H.: A class of iterative methods for solving saddle point problems. Numer. Math. 56, 645-666 (1990).

[3] Braess, D.: Finite elements. Theory, fast solvers, and applications in solid mechanics. Cambridge: University Press, 1997.

[4] Braess, D., Dahmen, W., Wieners, C.: A multigrid algorithm for the mortar finite element method. SIAM J. Numer. Anal. 37, 48-69 (1999).

[5] Braess, D., Dryja, M., Hackbusch, W.: A multigrid method for nonconforming fe-discretizations with application to non-matching grids. Computing 63, 1-25 (1999).

[6] Braess, D., Sarazin, R.: An efficient smoother for the Stokes problem. Appl. Numer. Math. 23, 3-19 (1997).

[7] Braess, D., Verfürth, R.: Multi-grid methods for nonconforming finite element methods. SIAM J. Numer. Anal. 27, 979-986 (1990).

[8] Brenner, S. C.: An optimal-order multigrid method for $P_{1}$ nonconforming finite elements. Math. Comp. 52, 1-15 (1989).

[9] Brenner, S. C.: A nonconforming multigrid method for the stationary Stokes equations. Math. Comp. 55, 411-437 (1990).

[10] Chen Z., Kwak, D. Y., Yoon J. Y.: Multigrid algorithms for nonconforming and mixed methods for nonsymmetric and indefinite problems. SIAM J. Sci. Comput. 19, 502-515 (1998).

[11] Chen Z., Oswald P.: Multigrid and multilevel methods for nonconforming $Q_{1}$ elements. Math. Comput. 67, 667-693 (1998).

[12] Crouzeix, M., Raviart, P. A.: Conforming and nonconforming finite element methods for solving the stationary Stokes equations I.R.A.I.R.O. Analyse Numerique $R$-3, 33-76 (1973).

[13] Hackbusch, W.: Multi-grid methods and applications. Berlin Heidelberg New York: Springer, 1985.

[14] Hestenes, M. R., Stiefel, E.: Methods of conjugate gradients for solving linear systems. J. Res. Nat. Bur. Standards 49, 409-436 (1952).

[15] John, V.: A comparison of parallel solvers for the incompressible Navier-Stokes equations. Comput. Visual. Sci. 4, 193-200 (1999).

[16] John, V., Tobiska, L.: Numerical performance of smoothers in coupled multigrid methods for the parallel solution of the incompressible Navier-Stokes equations. Int. J. Num. Meth. Fluids (to appear).

[17] Rannacher, R., St. Turek.: Simple nonconforming quadrilateral Stokes element. Numer. Meth. Part. Diff. Eq. 8, 97-111 (1992).

[18] Saad, Y., Schultz, M. H.: GMRES: A generalized minimal residual algorithm for solving nonsymmetric linear systems. SIAM J. Sci. Stat. Comput. 7, 856-869 (1986).

[19] Sarazin, R.: Eine Klasse von effizienten Glättern vom Jacobi-Typ für das Stokes-Problem PhD thesis, Ruhr-Universität Bochum, 1996.

[20] Schieweck, F.: Parallele Lösung der stationären inkompressiblen Navier-Stokes Gleichungen Otto-von-Guericke Universität Magdeburg, Fakultät für Mathematik, 1997. Habilitation.

[21] Turek, S.: Multigrid techniques for a divergence-free finite element discretization. East-West J. Numer. Math. 2, 229-255 (1994).

\author{
V. John \\ L. Tobiska \\ Otto-von-Guericke-Universität Magdeburg \\ Institut für Analysis und Numerik \\ Postfach 4120 \\ D-39016 Magdeburg \\ Germany \\ e-mail: john@mathematik.uni-magdeburg.de \\ tobiska@mathematik.uni-magdeburg.de
}

